random-effects model for meta-analysis, as this includes consideration of heterogeneity in the effect-size estimate. The authors also note that 'even though a random-effects model helps to consider heterogeneity, it does not remove it - heterogeneity still needs to be considered in interpreting the results. We used a random-effects model and examined heterogeneity.

We would like to reiterate that for those who wish to examine for themselves other points of the type raised by Peters, a detailed database of the studies we included is available online (http:// www.cbtinschizophrenia.com/).

1 Hunt N. How Science Takes Stock: The Story of Meta-Analysis. Sage, 1997.

2 White R, Gumley A, McTaggart J, Rattrie L, McConville D, Cleare S, et al. A feasibility study of Acceptance and Commitment Therapy for emotional dysfunction following psychosis. Behav Res Ther 2011; 49: 90-7.

3 Gaudiano BA, Herbert JD. Acute treatment of inpatients with psychotic symptoms using Acceptance and Commitment Therapy: pilot results. Behav Res Ther 2006; 44: 415-37.

4 Leclerc C, Lesage AD, Ricard N, Lecomte T, Cyr M. Assessment of a new rehabilitative coping skills module for persons with schizophrenia. Am J Orthopsychiatry 2000; 70: 380-8.

5 Penn DL, Meyer PS, Evans E, Wirth RJ, Cai K, Burchinal M. A randomized controlled trial of group cognitive-behavioral therapy $\mathrm{v}$. enhanced supportive therapy for auditory hallucinations. Schizophr Res 2009; 109: 522-9.

6 Shawyer F, Farhall J, Mackinnon A, Trauer T, Simms E, Ratcliff K, et al. A randomised controlled trial of acceptance-based cognitive behavioural therapy for command hallucinations in psychotic disorders. Behav Res Ther 2012; 50: 110-21.

7 Trower $\mathrm{P}$, Birchwood M, Meaden A, Byrne S, Nelson A, Ross $\mathrm{K}$ Cognitive therapy for command hallucinations: randomised controlled trial. Br J Psychiatry 2004; 184: 312-20.

8 Cochrane Consumers and Communication Review Group; Ryan R. Heterogeneity and Subgroup Analyses in Cochrane Consumers and Communication Review Group Reviews: Planning the Analysis at Protocol Stage. February 2014 (http://cccrg.cochrane.org).

P. J. McKenna, MB ChB, J. Radua, MD, PhD, FIDMAG Germanes Hospitalàrie Research Foundation and CIBERSAM, Spain. Email: mckennapeter1@googlemail.com; K. R. Laws, PhD, School of Life and Medical Sciences, University of Hertfordshire, UK; S. Jauhar, MB, ChB, BSC (Hons), Department of Psychosis Studies, Institute of Psychiatry, London, UK.

doi: 10.1192/bjp.205.2.160

\section{Borderline personality disorder and mood}

Parker examined whether borderline personality disorder (BPD) is a bipolar or unipolar mood condition and concluded by suggesting that it is probably neither. ${ }^{1}$ I would like to offer a supplementary interpretation of the literature; that is BPD is in large part a mood disorder but is not necessarily a bipolar or unipolar mood variant.

Borderline personality disorder is highly comorbid with bipolar disorder ${ }^{2}$ and depression, ${ }^{3}$ and those who develop bipolar disorder have early temperamental markers of emotional dysregulation. ${ }^{4}$ Support that BPD is a mood disorder is also aligned with the fact that affective instability is a core feature of the syndrome. While under- investigated, there is emerging evidence that affect or mood instability, as opposed to mood episodes, might be the core feature of bipolar disorders. ${ }^{5}$ The majority of patients with established bipolar disorder, even after symptomatic control continue to experience daily or weekly mood swings. ${ }^{6}$ Further, the prevalence of mood instability and cyclothymic temperament is increased in unaffected bipolar probands ${ }^{7}$ and it predicts functioning in those with bipolar disorder. ${ }^{5}$ Mood instability is highly prevalent in unipolar depression $^{8}$ and independently links to suicidality and healthservice use. Furthermore in BPD, affective instability is the least stable of the 'trait-like' features of the syndrome over 2 years.' Thus, all three disorders share mood instability as a clinical component and this all points to $\mathrm{BPD}$, at least in part, being a disorder of mood.

However BPD does not exactly fit into the bipolar or depressive affect rubric, given that the affective shifts do not last long enough for either diagnosis. Detailed studies of the nature of affective instability in mood disorders and BPD using the same measurement methods are limited. However, as Parker states, there are differences. Those with bipolar disorder have greater levels of euthymia-elation and affect intensity. In BPD there are more shifts between anxiety, depression and euthymia-anger. ${ }^{10}$ Negative emotionality is a critical feature of BPD but it is changeable, as is obvious to clinicians who have been charged with the care of people with BPD on in-patient wards.

Affect can be studied on the basis of intensity, frequency of shift, rapidity of rise-times and return to baseline, reactivity to psychosocial cues or whether endogenously driven, and the extent to which there is overdramatic expression. ${ }^{11}$ To this could be added valence. Using this framework, BPD could be conceptualised as a disorder of mood in which affect changes are intense, frequent, rapid to occur, slow to dissipate and in which the valence of the mood state is typically negative incorporating depression, anxiety and anger. This pattern of difficulties although related to mood, do not appear to overlap to a significant extent with how depression or bipolar disorder might be described using the same affective framework. Though it is clear that terms such as 'intensity', 'frequency' and 'rapidity of rise' need to be better specified, experience-sampling methods analysing affective patterns in the three disorders might further illuminate this area and indeed the debate.

1 Parker G. Is borderline personality disorder a mood disorder? $\mathrm{Br} J$ Psychiatry 2014; 204: 252-3.

2 Mantere O, Melartin TK, Suominen K, Rytsälä HJ, Valtonen HM, Arvilommi P, et al. Differences in Axis I and II comorbidity between bipolar I and II disorders and major depressive disorder. J Clin Psychiatry 2006; 67: 584-93.

3 Grant BF, Chou SP, Goldstein RB, Huang B, Stinson FS, Saha TD, et al. Prevalence, correlates, disability, and comorbidity of DSM-IV borderline personality disorder: results from the Wave 2 National Epidemiologic Survey on Alcohol and Related Conditions. J Clin Psychiatry 2008; 69: 533-45.

4 Luby JL, Navsaria N. Pediatric bipolar disorder: evidence for prodromal states and early markers. J Child Psychol Psychiatry 2010; 51: 459-71.

5 Strejilevich S, Martino D, Murru A, Teitelbaum J, Fassi G, Marengo E, et al. Mood instability and functional recovery in bipolar disorders. Acta Psychiatr Scand 2013; 128: 194-202.

6 Bonsall MB, Wallace-Hadrill SM, Geddes JR, Goodwin GM, Holmes EA. Nonlinear time-series approaches in characterizing mood stability and mood instability in bipolar disorder. Proc Biol SCi 2012; 279: 916-24.

7 Diler RS, Birmaher B, Axelson D, Obreja M, Monk K, Hickey MB, et al. Dimensional psychopathology in offspring of parents with bipolar disorder. Bipolar Disord 2011; 13: 670-8.

8 Marwaha S, Parsons N, Flanagan S, Broome M. The prevalence and clinical associations of mood instability in adults living in England: results from the Adult Psychiatric Morbidity Survey 2007. Psychiatry Res 2013; 205: 262-8.

9 Chanen AM, Jackson HJ, McGorry PD, Allot KA, Clarkson V, Yuen HP. Two-year stability of personality disorder in older adolescent outpatients. J Pers Disord 204; 18: 526-41.

10 Reich DB, Zanarini MC, Fitzmaurice G. Affective lability in bipolar disorder and borderline personality disorder. Compr Psychiatry 2013; 53: 230-7.

11 Koenigsberg HW. Affective instability: toward an integration of neuroscience and psychological perspectives. J Pers Disord 2010; 24: 60-82.

Steven Marwaha, Associate Clinical Professor of Psychiatry and Consultant Psychiatrist, Division of Mental Health and Wellbeing, Warwick Medical School, University of Warwick, UK. Email: s.marwaha@warwick.ac.uk.

doi: 10.1192/bjp.205.2.161

Gordon Parker makes a powerful case against the hypothesis that borderline personality disorder is really a form of bipolar or unipolar disorder. ${ }^{1}$ In so doing he is tilting at a windmill in whose 